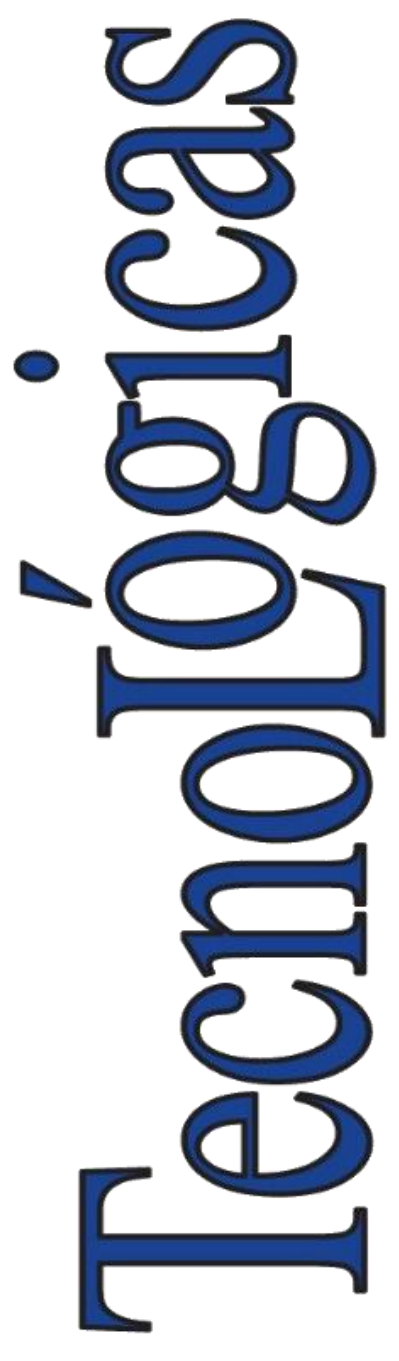

ISSN-p: 0123-7799 ISSN-e: 2256-5337

Vol. 24, nro. 52, e2113, 2021

Recibido: 3 agosto 2021 Aceptado: 18 noviembre 2021 Disponible: 16 diciembre 2021

CInstituto Tecnológico Metropolitano Este trabajo está licenciado bajo una Licencia Internacional Creative Commons Atribución (CCBY-NC-SA)

\section{Propiedades físicas de productos cocristalizados de jugo de maracuyá y pulpa de guayaba y su cinética de cocristalización}

\section{Physical Properties of Co-crystallized Products of Passion Fruit (Passiflora Edulis) Juice and Guava (Psidium Guajava I) Pulp and Their Co-Crystallization Kinetics}

\author{
(D) Ramiro Torres-Gallo ${ }^{1}$; \\ (D) Teófilo Arteaga-Cavadia ${ }^{2}$; \\ iD Everaldo Montes-Montes ${ }^{3}$;
}

Cómo citar / How to cite

R. Torres-Gallo; T. Arteaga-Cavadia; E. Montes-Montes, "Propiedades físicas de productos cocristalizados de jugo de maracuyá y pulpa de guayaba y su cinética de cocristalización”, TecnoLógicas, vol. 24, nro. 52, e2113, 2021. https://doi.org/10.22430/22565337.2113

${ }^{1}$ Universidad del Atlántico, Barranquilla-Colombia, ramirotorres@mail.uniatlantico.edu.co 2 Universidad de Córdoba, Montería-Colombia, tarteaga@correo.unicordoba.edu.co

${ }^{3}$ Universidad de Córdoba, Montería-Colombia, everaldomontes@correo.unicordoba.edu.co 


\section{Resumen}

La cocristalización con sacarosa se ha utilizado para obtener productos con mejores propiedades físicas, como solubilidad, humectabilidad, hidratación y estabilidad, así como para preservar los componentes activos presentes en la mayoría de las futas. El objetivo de este trabajo fue determinar el efecto del $\mathrm{pH}$ y concentración de pulpa de guayaba (Psidium guajava L) y jugo de maracuyá (Passiflora edulis, en adelante concentración de fruta), sobre la humedad, la densidad aparente, la solubilidad, el ángulo de reposo, la higroscopicidad y la cinética de cocristalización de productos cocristalizados de jugo de maracuyá y pulpa de guayaba. Se prepararon los cocristalizados con jarabe de sacarosa a $70{ }^{\circ}$ Brix, pulpa de guayaba concentrada a $18^{\circ}$ Brix y el jugo de maracuyá a $40^{\circ}$ Brix. Para concentrar las mezclas y prepararlas para la cocristalización se utilizó un evaporador rotativo Heidolph D-91126 HB y un controlador de vacío Heidolph $\mathrm{DC} 1 / 1 \mathrm{HO}$. La cinética de cocristalización se evaluó utilizando un reómetro TA Instrument AR 1500ex, determinando la viscosidad compleja en el tiempo, a través de una rampa de temperatura de $80^{\circ} \mathrm{C}$ a $15{ }^{\circ} \mathrm{C}$ a $1 \mathrm{~Hz}$, descendiendo $6,5^{\circ} \mathrm{C} / \mathrm{min}$, ajustando los datos experimentales a un modelo empírico. El aumentar la concentración de fruta añadida en el proceso, aumentan el tiempo de cristalización y la humedad final del producto, y disminuye su solubilidad. La velocidad de cocristalización se ve favorecida por la menor concentración de fruta, presentándose una mayor velocidad de cristalización para los cocristalizados de pulpa de guayaba que en los de jugo de maracuyá.

\section{Palabras clave}

Sacarosa, microencapsulación, jugo de fruta, alimentos en polvo, cocristalización.

\section{Abstract}

Co-crystallization with sucrose has been used to obtain products with better physical properties (such as solubility, wettability, hydration, and stability) and to preserve the active components present in most fruits. The objective of this study was to determine the effect of the $\mathrm{pH}$ and concentration of guava (Psidium guajava L) pulp and passion fruit (Passiflora edulis) juice (both referred to as fruit concentration) on the moisture, apparent density, solubility, angle of repose, hygroscopicity, and co-crystallization kinetics of co-crystallized passion fruit juice and guava pulp products. The co-crystallized products were prepared with sucrose syrup at $70{ }^{\circ}$ Brix, concentrated guava pulp at $18^{\circ}$ Brix, and passion fruit juice at $40^{\circ}$ Brix. A Heidolph D-91126 HB Rotary-evaporator and a Heidolph DC1/1HO Vacuum Controller were used to concentrate the mixtures and prepare them for co-crystallization. The co-crystallization kinetics was evaluated using a TA Instrument AR 1500ex rheometer, thus determining complex viscosity over time using a temperature ramp from 80 to $15^{\circ} \mathrm{C}$ at $1 \mathrm{~Hz}$, descending at $6.5^{\circ} \mathrm{C} / \mathrm{min}$. Then, the experimental data were fitted to an empirical model. Our results show that increasing the fruit concentration added in the process extends the crystallization time, increases the final moisture of the product, and decreases its solubility. The co-crystallization rate was favored by the lowest fruit concentration, and a higher crystallization rate was observed in the guava pulp than in the passion fruit juice (two cocrystallized products).

\section{Keywords}

Sucrose, Microencapsulation, Fruit juice, Food powders, Co-crystallization. 


\section{INTRODUCCIÓN}

Las frutas son fuente de compuesto bioactivo como carotenoides minerales, vitamina A, complejos de vitamina $\mathrm{B}$ y vitamina $\mathrm{C}$, los cuales están asociados a la prevención de múltiples enfermedades, ya que actúan como antiinflamatorios, anticancerígenos, antidiabéticos y previenen enfermedades cardiovasculares y coronarias [1]. De los frutos tropicales destacados como fuentes relevantes de compuestos bioactivos está el maracuyá (Passiflora edulis) y la guayaba (Psidium guajava L) [2], [3]. Sin embargo, los compuestos bioactivos presentes en las frutas suelen oxidarse fácilmente durante el procesamiento o almacenamiento por ser muy sensibles a la temperatura, la luz, los cambios de $\mathrm{pH}$ y los iones metálicos, entre otros [4], [5].

La degradación de compuesto bioactivo, durante el procesamiento de las frutas, ha sido reportado en diversas investigaciones, así como el comportamiento cinético de esta degradación, de aquí la importancia de evaluar métodos de procesamientos que permitan conservar este importante compuesto [6], [7]. Hay varios métodos de microencapsulación reportados en literatura [8], [9]. Sin embargo, la microencapsulación por cocristalización es un método simple que ofrece una alternativa económica para la incorporación del ingrediente activo, obteniéndose un producto en polvo, lo que resulta en una facilidad de manejo y, al mismo tiempo, preserva los componentes activos [8], [10].

La cocristalización con sacarosa se ha utilizado para producir una forma más apetecible que enmascara sabor amargo de los agentes activos con el zinc, obteniéndose productos que proporcionan ventajas significativas en las industrias farmacéutica y de dulces [11]. También se han reportado estudios que indican un alto rendimiento de atrapamiento de antioxidantes en extractos de vegetales obteniéndose productos con alta estabilidad y mejorando propiedades como actividad del agua, contenido de humedad, higroscopicidad y fluidez [12], [13]. Por otra parte, la cocristalización de antioxidantes naturales con sacarosa ha permitido obtener comprimidos con bajos tiempos de desintegración y una liberación rápida en medio acuoso [14].

Durante la cocristalización, la sacarosa, que es una estructura cristalina sólida, se aglomera en cristales irregulares de tamaño micro, con espacios vacíos y mayor superficie, proporcionando una base porosa para la incorporación del componente activo, de manera que en la solución sobresaturada se cristaliza simultáneamente ambos componentes. El proceso de cocristalización implica la incorporación de un ingrediente activo en microcristales de sacarosa por cristalización espontánea [15]. Al incorporar cuidadosamente el ingrediente activo en la sacarosa se previene la cristalización prematura de ésta, permitiendo que el proceso se realice a velocidades reproducibles [16], obteniéndose un producto cocristalizado con mejores propiedades físicas, tales como solubilidad, la humectabilidad, la hidratación, la dispersión, las propiedades antiapelmazantes y la estabilidad de los materiales encapsulados [17], [18]. Sin embargo, el exceso de material activo en relación con el agente encapsulador y el $\mathrm{pH}$ de los extractos en proceso de encapsulamiento, puede afectar la velocidad de cristalización, teniendo efectos negativos sobre las propiedades del producto resultante [16], [19]-[21].

El objetivo de este trabajo fue estudiar la influencia del pH y concentración de fruta en el proceso de cocristalizado de jugo de maracuyá (Passiflora edulis) y pulpa guayaba (Psidium guajava $L$ ), sobre las propiedades físicas y en la cinética de cristalización del producto. 


\section{METODOLOGíA}

\subsection{Materia prima}

Se utilizaron guayabas (Psidium guajava L.) variedad híbrida de Klom Sali, y maracuyás (Passiflora edulis) obtenidas de un lote único, procedente de Cereté (Colombia). Las frutas se seleccionaron en estado de madures comercial. Las guayabas (Psidium guajava L.) se seleccionaron en estado de madures 6, según la escala de color propuesta por [22] de forma aleatoria; y las maracuyás (Passiflora edulis), se seleccionaron en escala de color 3, propuesta por [23]. La sacarosa refinada, al 99,8 \% de pureza tipo comercial, se utilizó como una matriz encapsulante. La pulpa de la guayaba se obtuvo en una despulpadora con un tamiz de malla $1.5 \mathrm{~mm}$. El jugo de maracuyá de forma manual, utilizando una licuadora y colador comercial. La pulpa y el jugo se empacaron en bolsas herméticas y se refrigeraron a $4{ }^{\circ} \mathrm{C}$.

\subsection{Análisis físicos y químicos de la pulpa y jugo}

A la pulpa de guayaba y el jugo de maracuyá se les realizaron pruebas de $\mathrm{pH}$, según método de la AOAC10,041/84, ${ }^{\circ}$ Brix con refractómetro marca MISCO ${ }^{\circledR}$ modelo $1043 \mathrm{vp}$, la acidez titulable, según método AOAC 31,231/ 84 y el índice de madurez se obtuvo de la relación de los sólidos solubles totales y la acidez.

\subsection{Encapsulamiento}

Se preparó un jarabe de sacarosa a $70{ }^{\circ}$ Brix, con agua desionizada, y se calentó hasta $120{ }^{\circ} \mathrm{C}$ en una plancha de calentamiento con agitación magnética MS-H280-Pro ${ }^{\circledR}$ hasta sobresaturación ( $83^{\circ}$ Brix) para la cristalización espontánea [15].

La pulpa de guayaba se concentró hasta $18^{\circ}$ Brix y el jugo de maracuyá a $40{ }^{\circ}$ Brix, utilizando el rotoevaporador Heidolph D-91126 HB digital ${ }^{\circledR}\left(\right.$ a $\left.30^{\circ} \mathrm{C}\right)$, ajustando el $\mathrm{pH}(3,0$, 4,0 y 5,0 según diseño de experimento) con hidróxido de calcio $4 \mathrm{~N}$ y ácido cítrico, grado alimenticio, si era necesario. El pH se midió con un $\mathrm{pH}$-metro digital Mettler Toledo ${ }^{\circledR}$, según el método de la AOAC 10,041/84. Las concentraciones en ${ }^{\circ}$ Brix se determinaron con refractómetro marca MISCO ${ }^{\circledR}$ modelo $10431 \mathrm{vp}$.

$\mathrm{Al}$ jarabe de sacarosa, a $70{ }^{\circ}$ Brix y $120{ }^{\circ} \mathrm{C}$, se le adicionó la pulpa de guayaba y jugo de maracuyá concentrado (10\%, $20 \%$ y $30 \%$ ), según diseño de experimento, por separado. Las mezclas se agitaron en un homogeneizador Ultra-Turrax T-50 Basic ${ }^{\circledR}$ a 3000 rpm hasta $40,0 \pm 0,5^{\circ} \mathrm{C}$, donde se obtuvieron los respectivos cocristalizados de pulpa de guayaba y jugo de maracuyá. Posteriormente, se secaron en una estufa al vacío Thermo Scientific a $40,0 \pm 0,5^{\circ} \mathrm{C}$ durante 24 horas aproximadamente, se realizó una molienda y un tamizado (malla 20 de la serie de tamices de Tyler), se empacaron en bolsas de cierre fácil marca Ziplock a $30^{\circ} \mathrm{C}$ y se almacenaron en un desecador.

\subsection{Caracterización física de los cocristalizados}

A las muestras de los respectivos cocristalizados se les determinó la humedad, en base húmeda, en una balanza de luz halógena Moisture Analyzer ${ }^{\circledR}$, según metodología de [24]; la densidad aparente, la solubilidad, medida como el tiempo necesario para una completa disolución de $10 \mathrm{~g}$ de producto en $100 \mathrm{~mL}$ de agua destilada a $25^{\circ} \mathrm{C}$, y el ángulo de reposo se determinaron según la metodología propuesta por [17]; la higroscopicidad se determinó según metodología propuesta por [25], Para ello, se pesó aproximadamente $1 \mathrm{~g}$ de cada muestra de 
polvo en cajas de Petri, colocándose a $25^{\circ} \mathrm{C}$ en un recipiente hermético de vidrio lleno de solución saturada de cloruro de sodio. Las muestras se pesaron durante una semana y se expresó como gramos de humedad por 100 g de sólidos.

\subsection{Cinética de cristalización}

Para determinar la cinética de cristalización se tomaron muestras de la mezcla de solución de sacarosa con concentraciones de fruta de $10 \%, 20 \%$ y $30 \%$ y se llevó al reómetro TA Instrument AR $1500 \mathrm{ex}$, donde se realizó una rampa de temperatura desde $80{ }^{\circ} \mathrm{C}$ a $15^{\circ} \mathrm{C}$ a $1 \mathrm{~Hz}$ de frecuencia y torque de 10 micro $\mathrm{Nm}$, descendiendo $6,5^{\circ} \mathrm{C} / \mathrm{min}$, obteniéndose valores de viscosidad compleja vs tiempo.

Los datos experimentales de viscosidad compleja se ajustaron al modelo matemático (1) propuesto por [26] para representar la viscosidad compleja a lo largo del proceso de cristalización de un sistema, que representa el comportamiento cinético de cristalización.

$$
\frac{\left|\eta^{*}\right|}{\left|\eta^{*}\right|_{0}}=(1+\propto t)^{\beta}
$$

Donde $\left|\eta^{*}\right|$ es la viscosidad compleja del sistema formado por la solución sobresaturada y los microcristales, $\left|\eta^{*}\right|_{0}$ es la viscosidad compleja inicial del sistema, que corresponde a la solución de sacarosa sobresaturada, ya mezclada al jugo concentrado, $t$ es el tiempo de cristalización y a y $B$ son parámetros empíricos de [26].

\subsection{Diseño de experimento y análisis estadístico}

El experimento se condujo bajo un diseño completamente al azar con arreglo factorial $3^{2}$ para los factores $\mathrm{pH}(3,0,4,0$ y 5,0$)$ y contenido de fruta en la mezcla $(10 \%, 20 \%$ y $30 \%)$ para las variables de respuesta: características fisicoquímicas del cocristalizado. Para determinar la influencia de los factores sobre las variables de respuesta, se empleó el procedimiento de modelo lineal generalizado (GLM), que permite construir un modelo estadístico que describe el impacto de uno o más factores en una o más variables dependientes y a partir del modelo poder optimizar la variable de respuesta, en el rango de factores estudiados. El análisis de los resultados se realizó en el software Statgraphics Centurion XV®, con un análisis de varianza (nivel de confianza será del $95 \%$ y un análisis de gráficos de superficie de respuesta que arrojó el modelo. El criterio de evaluación de ajuste de modelo en la evaluación de la cinética de cristalización fue el coeficiente de determinación y el análisis de residuales.

\section{RESULTADOS Y DISCUSIÓN}

\subsection{Análisis físicos y químicos de la pulpa y jugo}

Los valores de $\mathrm{pH}$, sólidos solubles ( ${ }^{\circ}$ Brix) obtenidos en las pulpas de guayaba (Tabla 1), coinciden con los valores reportado por [27]-[29]. Sin embargo, presenta valores por encima de los rangos de acidez titulable para guayaba Criolla Roja, reportado por [30]. De igual manera los valores de $\mathrm{pH}$, sólidos solubles ( ${ }^{\circ}$ Brix) del jugo de maracuyá coinciden con los valores reportado por [31]-[33], Sin embargo, la acidez titulable está por encima de los valores reportado por este último. Este comportamiento puede explicarse, según [34], porque la acidez 
puede cambiar con el cultivar y con la época del año, mientras que los valores de $\mathrm{pH}$ y solidos solubles ( ${ }^{\circ}$ Brix) son mayormente afectadas por el estado de madurez.

Tabla 1. Propiedades químicas de las pulpas de guayaba y jugo de maracuyá. Fuente: elaboración propia.

\begin{tabular}{ccc}
\hline Parámetro & Guayaba & Maracuyá \\
\hline $\mathrm{pH}$ & $4,02 \pm 0,11$ & $3,05 \pm 0,03$ \\
Sólidos Solubles totales ( ${ }^{\circ}$ Brix) & $8,70 \pm 0,62$ & $15,91 \pm 0,56$ \\
Acidez Titulable (\% ácido cítrico) & $0,79 \pm 0,05$ & $4,31 \pm 0,01$ \\
índice de madurez (Sólidos Solubles totales /Acidez Titulable) & $11,01 \pm 1,02$ & $3,96 \pm 0,38$ \\
\hline
\end{tabular}

\subsection{Caracterizaciones físicas de los cocristalizados}

Humedad: El análisis de varianza (ANOVA) para la humedad de cocristalizado de pulpa de guayaba (Hg) y de jugo de maracuyá (Hm) (Tabla 2), muestra que la interacción no presentó diferencia significativa (con un nivel de confianza del 95,0\%), al igual que los efectos principales y cuadrático de $\mathrm{pH}$. Consecuentemente, se considera eliminar este factor de los modelos estadístico. Los estadísticos $\mathrm{r}^{2}$ indican que el modelo así ajustado explica 98,91 \% y 92,73 \% la variabilidad de la humedad del cocristalizado de pulpa de guayaba y de jugo de maracuyá, respectivamente. Por tanto, la humedad de los cocristalizados depende sólo del contenido de fruta, ejerciendo el efecto cuadrático de la fruta una mayor influencia sobre la humedad del cocristalizado que los efectos principales.

Tabla 2. Análisis de varianza, modelos estadísticos y $\mathrm{r}^{2}$ para humedad de los productos cocristalizados Fuente: elaboración propia.

\begin{tabular}{|c|c|c|c|c|c|c|c|c|c|}
\hline \multirow[t]{2}{*}{ Fuente } & \multirow[t]{2}{*}{$\mathrm{Gl}$} & \multicolumn{2}{|c|}{ Suma de cuadrados } & \multicolumn{2}{|c|}{ Cuadrado medio } & \multicolumn{2}{|c|}{ Razón-F } & \multicolumn{2}{|c|}{ Valor-p } \\
\hline & & Guayaba & Maracuyá & Guayaba & Maracuyá & Guayaba & Maracuyá & Guayaba & Maracuyá \\
\hline $\mathrm{pH}$ & 1 & 0,0002 & 0,0050 & 0,0002 & 0,0050 & 0,06 & 3,40 & 0,8079 & 0,0792 \\
\hline [Fruta $]^{*}$ & 1 & 5,8939 & 0,4544 & 5,8939 & 0,4544 & 1786,60 & 309,39 & 0,0000 & 0,0000 \\
\hline $\mathrm{pH}^{*} \mathrm{pH}$ & 1 & 0,0003 & 0,0000 & 0,0003 & 0,0000 & 0,08 & 0,00 & 0,7790 & 1,0000 \\
\hline$[$ Fruta $] *[$ Fruta $]$ & 1 & 0,6801 & 0,0043 & 0,6801 & 0,0043 & 206,15 & 2,90 & 0,0000 & 0,1031 \\
\hline $\mathrm{pH}^{*}[$ Fruta $]$ & 1 & 0,0027 & 0,0001 & 0,0027 & 0,0001 & 0,82 & 0,09 & 0,3759 & 0,7662 \\
\hline Residuo & 21 & 0,0693 & 0,0308 & 0,0033 & 0,0015 & & & & \\
\hline $\begin{array}{l}\text { Total } \\
\text { (corregido) }\end{array}$ & 26 & 6,6464 & 0,4947 & & & & & & \\
\hline $\begin{array}{l}\text { Modelo } \\
\text { ajustado* }\end{array}$ & \multicolumn{5}{|c|}{$\mathrm{H}_{\mathrm{g}}=2,378-0,072[$ Fruta $]+0,003[\text { Fruta }]^{2}$} & \multicolumn{4}{|c|}{$\mathrm{H}_{\mathrm{m}}=1,505-0,026[$ Fruta $]+0,001[\text { Fruta }]^{2}$} \\
\hline $\mathrm{r}^{2}$ & \multicolumn{5}{|c|}{$99,91 \%$} & \multicolumn{4}{|c|}{$92,73 \%$} \\
\hline
\end{tabular}

*[Fruta] representa contenido de pulpa de guayaba o jugo de maracuyá.

La humedad de cocristalizado de guayabas aumentó con la concentración de fruta (Figura 1) de forma exponencial, mientras el cocristalizado de maracuyá presenta un comportamiento casi lineal, por lo cual la humedad del cocristalizado de este último, aumento en menor proporción con la concentración de fruta. 


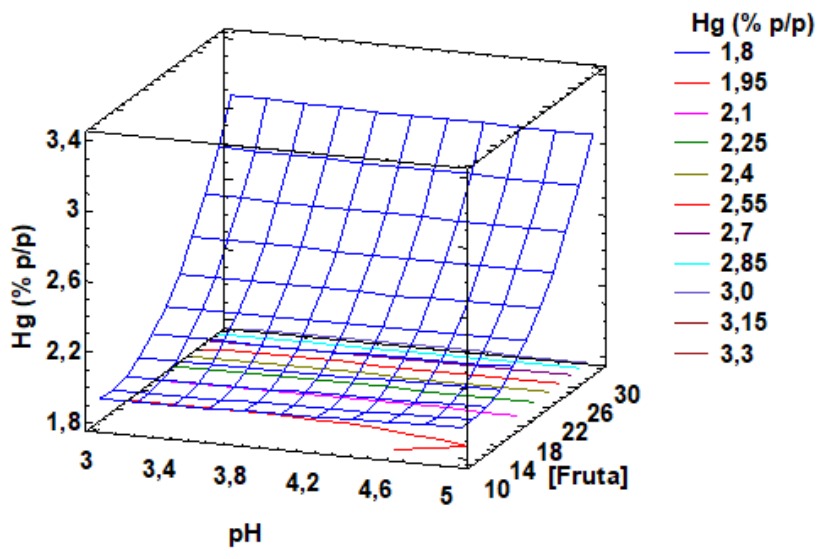

(a)

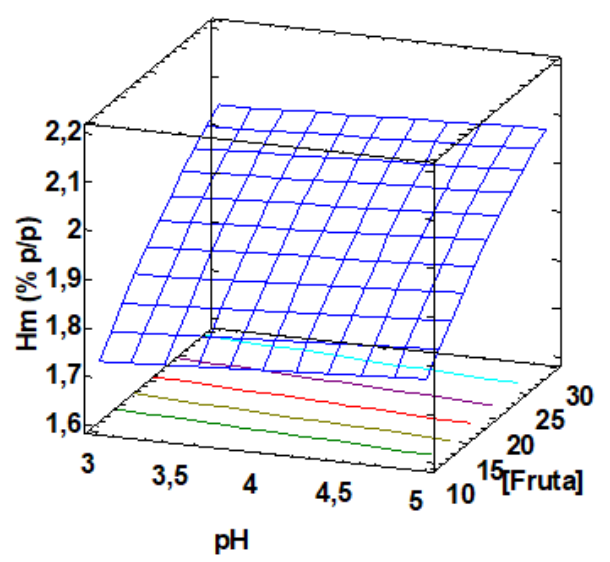

(b)

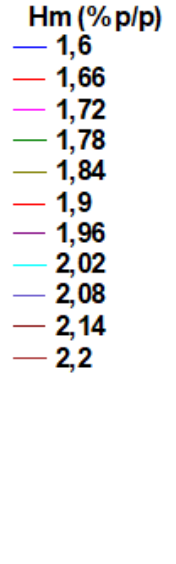

Figura 1. Humedad del cocristalizado. a. en función del pH y \% de pulpa de guayaba (Hg) (a) y jugo de maracuyá. Fuente: elaboración propia.

Esto puede estar relacionado con la disminución de la formación de conglomerados de mayor porosidad, en el cocristalizados de maracuyá, debido a mayores contenidos de sólidos solubles, lo cual desfavorecen la pérdida de humedad de los cocristales durante el proceso de secado [35]. La menor humedad del cocristalizado de guayaba, que se puede obtener según el modelo, fue de $1,93 \% \mathrm{p} / \mathrm{p}$ a $\mathrm{pH}$ de 3,0 y 11,5 \% de pulpa, y la de cocristalizado de jugo de maracuyá $(\mathrm{Hm})$ fue de $1,72 \% \mathrm{p} / \mathrm{p}$ a $\mathrm{pH}$ de 3,0 y $10,0 \%$ de jugo.

El rango de humedad de cocristalizado de pulpa de guayaba $(1,91 \pm 0,07 \%-3,10 \pm 0,06 \%$ $\mathrm{p} / \mathrm{p}$ y el de jugo de maracuyá $(1,72 \pm 0,04 \%-2,08 \pm 0,08 \%)$ p/p, coinciden con los cocristalizados de naranja agria $(2,5 \%-4,5 \%) \mathrm{p} / \mathrm{p}$ [35] y encapsulamiento por aspersión de un producto a base de lactosuero y pulpa de mango $(1,58 \%-5,84 \%) \mathrm{p} / \mathrm{p}$ [36]. No obstante, se reportaron valores más alto en jugo de naranja secado por aspersión $(4,53 \pm 0,01 \%-5,36 \pm$ $0,01 \%) \mathrm{p} / \mathrm{p}$ [37] y valores entre (4,17\% a 8,24\%) p/p en microencapsulado de chirimoya [38].

Densidad aparente: El ANOVA para la densidad aparente del cocristalizado de pulpa de guayaba (Dg) y de jugo de maracuyá (Dm) (Tabla 3) muestran que la interacción al igual que los efectos principal y cuadrático de $\mathrm{pH}$ no resultaron significativo (con un nivel de confianza del 95,0 \%). Consecuentemente, se considera eliminar este factor de los modelos estadístico. Los estadísticos $\mathrm{r}^{2}$ indican que el modelo así ajustado explica en un 86,23\% y 99,32 \% la variabilidad de la densidad aparente del cocristalizado de pulpa de guayaba y de jugo de maracuyá, respectivamente. Por tanto, la densidad aparente de los cocristalizados depende sólo de la concentración de fruta, ejerciendo el efecto cuadrático de la fruta una mayor influencia sobre la densidad aparente del cocristalizado que los efectos principales (Figura 2). 
Tabla 3. Análisis de varianza para densidad aparente de los productos cocristalizados Fuente: elaboración propia.

\begin{tabular}{|c|c|c|c|c|c|c|c|c|c|}
\hline \multirow[t]{2}{*}{ Fuente } & \multicolumn{3}{|c|}{ Gl Suma de Cuadrados } & \multicolumn{2}{|c|}{ Cuadrado Medio } & \multicolumn{2}{|c|}{ Razón-F } & \multicolumn{2}{|c|}{ Valor-p } \\
\hline & & Guayaba & Maracuyá & Guayaba & Maracuyá & Guayaba & Maracuyá & Guayaba & Maracuyá \\
\hline $\mathrm{pH}$ & 1 & 0,0008 & 0,0001 & 0,0008 & 0,0001 & 1,06 & 0,28 & 0,3149 & 0,6014 \\
\hline [Fruta $]^{*}$ & 1 & 0,1034 & 0,0885 & 0,1034 & 0,0885 & 129,41 & 3060,48 & 0,0000 & 0,0000 \\
\hline $\mathrm{pH} \mathrm{H}^{*} \mathrm{pH}$ & 1 & 0,0003 & 0,0001 & 0,0003 & 0,0001 & 0,34 & 0,04 & 0,5655 & 0,8513 \\
\hline$[$ Fruta $] *[$ Fruta $]$ & 1 & 0,0127 & 0,0056 & 0,0127 & 0,0056 & 15,96 & 192,82 & 0,0007 & 0,0000 \\
\hline $\mathrm{pH}^{*}[$ Fruta $]$ & 1 & 0,0006 & 0,0001 & 0,0006 & 0,0001 & 0,81 & 1,06 & 0,3769 & 0,3144 \\
\hline Residuo & 21 & 0,0169 & 0,0006 & 0,0008 & 0,0001 & & & & \\
\hline $\begin{array}{l}\text { Total } \\
\text { (corregido) }\end{array}$ & 26 & 0,1347 & 0,0947 & & & & & & \\
\hline $\begin{array}{l}\text { Modelo } \\
\text { Ajustado* }\end{array}$ & \multicolumn{5}{|c|}{$\mathrm{D}_{\mathrm{g}}=0,687+0,011^{*}[$ Fruta $]-0,001 *[\text { Fruta }]^{2}$} & \multicolumn{4}{|c|}{$\mathrm{D}_{\mathrm{m}}=0,624+0,019 *[$ Fruta $]-0,001 *[\text { Fruta }]^{2}$} \\
\hline $\mathrm{r}^{2}$ & \multicolumn{5}{|c|}{$86,23 \%$} & \multicolumn{4}{|c|}{$99,32 \%$} \\
\hline
\end{tabular}

*[Fruta] representa contenido de pulpa de guayaba o jugo de maracuyá;

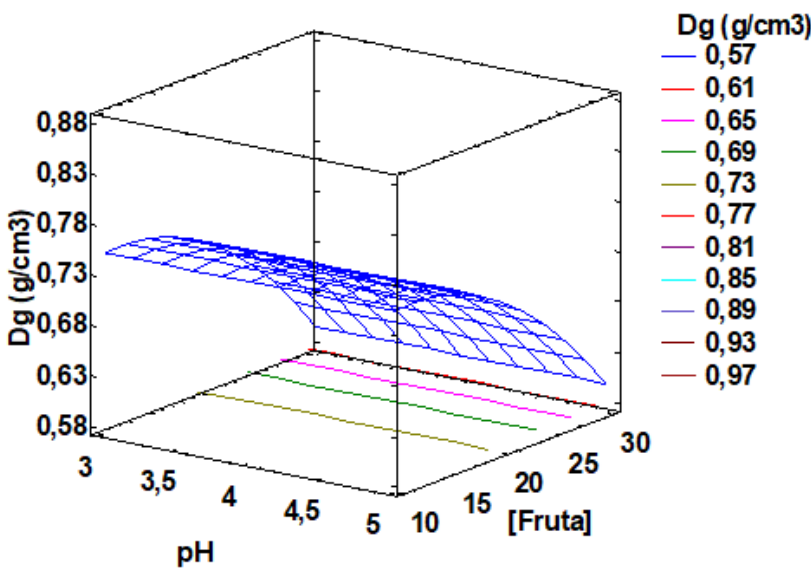

(a)

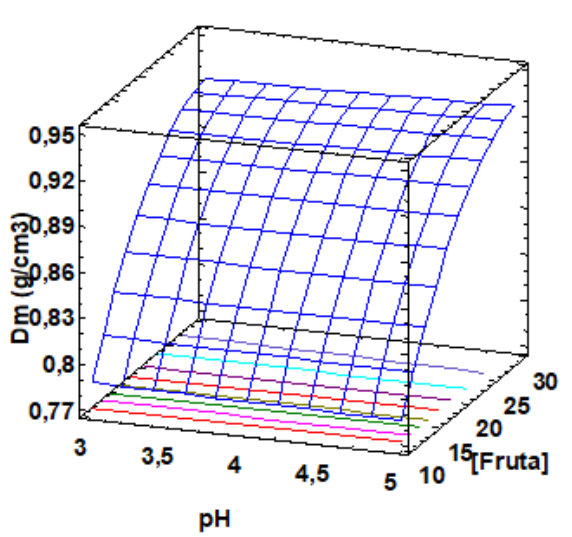

(b)

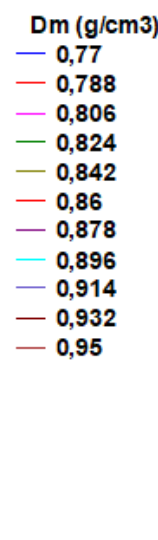

Figura 2. Densidad aparente del cocristalizado. en función del pH y \% de pulpa de guayaba (Dg) (a) y jugo de maracuyá (Dm) (b). Fuente: elaboración propia.

El rango de densidad aparente de cocristalizado de pulpa de guayaba $\left(0,59 \pm 0,01 \mathrm{~g} / \mathrm{cm}^{3}\right.$ $0,78 \pm 0,07 \mathrm{~g} / \mathrm{cm}^{3}$ ) coincide con el reportado para el cocristalizado de maracuyá comercial [39], a $64{ }^{\circ}$ Brix $(0,64-0,77) \mathrm{g} / \mathrm{cm}^{3}$; lo reportado para bebidas de cacao en polvo $(0,49-0,81) \mathrm{g} / \mathrm{cm}^{3}$ [40]; lo reportado para un suplemento alimenticio de alto contenido proteico $\left(0,463 \mathrm{~g} / \mathrm{cm}^{3}\right.$ $\left.0,526 \mathrm{~g} / \mathrm{cm}^{3}\right)$ [41] y un polvo para flan con fibra dietética de nopal $(0,59 \pm 0,01) \mathrm{g} / \mathrm{cm}^{3}$ [42], encontrándose generalmente en la franja correspondiente a la mayoría de los polvos alimenticios de $0,3 \mathrm{~g} / \mathrm{cm}^{3}$ a $0,8 \mathrm{~g} / \mathrm{cm}^{3}$ [43]. Sin embargo, los valores de densidad aparente de cocristalizado dejugo de maracuyá $\left(0,78 \pm 0,00 \mathrm{~g} / \mathrm{cm}^{3}-0,93 \pm 0,01 \mathrm{~g} / \mathrm{cm}^{3}\right)$, a concentración de jugo por encina de $10 \% \mathrm{p} / \mathrm{p}$, estuvieron por encima de esa franja.

La densidad aparente del cocristalizado de guayaba, para los rangos estudiados, disminuyó con la concentración de pulpa (Figura 2a), obteniéndose la mayor densidad aparente del cocristalizado de guayaba, según el modelo, en $0,75 \mathrm{~g} / \mathrm{cm}^{3}$, a pH de 4,1\% y $11,8 \%$ de pulpa. Esta disminución de la densidad aparente parece estar relacionada con la particularidad de la pulpa de promover la unión de partículas, unas con otras, de manera aleatoria, resultando un agregado de estructura abierta y porosa de mayor tamaño que las partículas originales [44]. Sin embargo, la densidad aparente del cocristalizado de maracuyá, 
presentó un comportamiento diferente, aumentando la densidad aparente con la concentración de jugo (Figura 2b), obteniéndose la mayor densidad aparente del cocristalizado de maracuyá, según el modelo, en $0,93 \mathrm{~g} / \mathrm{cm}^{3}$, a $\mathrm{pH}$ de 3,00 y $30,0 \%$ de jugo,

La mayor densidad aparente del cocristalizado de maracuyá respecto al cocristalizado de guayaba se puede atribuir a la mejor incorporación del azúcar en el jugo de maracuyá que en la pulpa de guayaba, pues al ser el cristalizado de maracuyá menos amorfo (Figura 3b), que el cocristalizado de guayaba (Figura 3a), evidencia que la sacarosa cristalizó mejor, fue más compacta y formó caras más planas, obteniéndose un producto menos poroso.

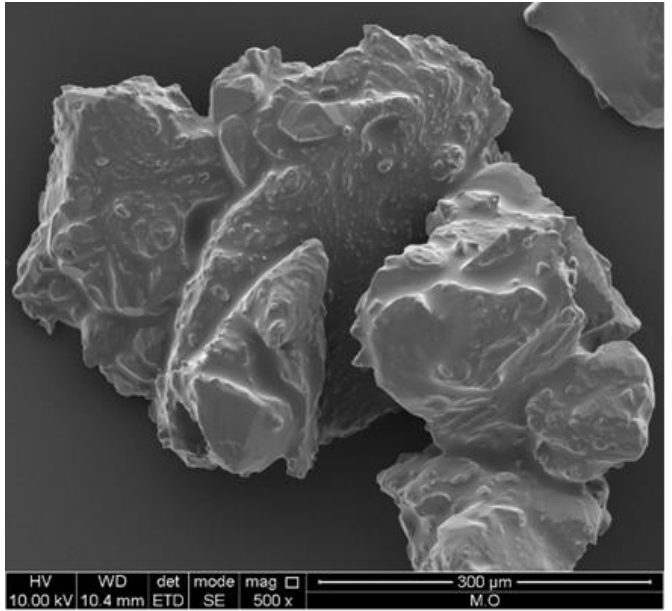

(a)

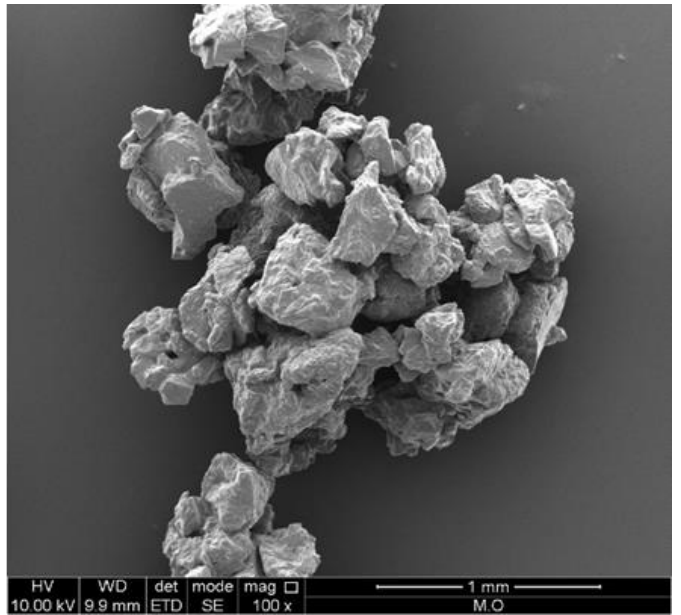

(b)

Figura 3. Microscopía electrónica de barrido de cocristalizado de pulpa de guayaba (a) y jugo de maracuyá (b). Fuente: elaboración propia.

Solubilidad: El ANOVA para la solubilidad de cocristalizado de pulpa de guayaba (Sg) y de jugo de maracuyá (Sm) (Tabla 4), muestra que la interacción no presentó diferencia significativa (con un nivel de confianza del 95,0\%), al igual que los efectos principales y cuadrático de $\mathrm{pH}$. Consecuentemente, se considera eliminar este factor de los modelos estadístico.

Tabla 4. Análisis de varianza para Solubilidad de los productos cocristalizados. Fuente: elaboración propia.

\begin{tabular}{|c|c|c|c|c|c|c|c|c|c|}
\hline \multirow[t]{2}{*}{ Fuente } & \multirow[t]{2}{*}{$\mathrm{Gl}$} & \multicolumn{2}{|c|}{ Suma de Cuadrados } & \multicolumn{2}{|c|}{ Cuadrado Medio } & \multicolumn{2}{|c|}{ Razón-F } & \multicolumn{2}{|c|}{ Valor-p } \\
\hline & & Guayaba & Maracuyá & Guayaba & Maracuyá & Guayaba & Maracuyá & Guayaba & Maracuyá \\
\hline $\mathrm{pH}$ & 1 & 0,056 & 10,89 & 0,056 & 10,89 & 0,01 & 1,61 & 0,9148 & 0,2184 \\
\hline [Fruta]* & 1 & 355,560 & 2005,56 & 355,560 & 2005,56 & 75,12 & 296,46 & 0,0000 & 0,0000 \\
\hline $\mathrm{pH}^{*} \mathrm{pH}$ & 1 & 3,130 & 0,07 & 3,130 & 0,07 & 0,66 & 0,01 & 0,4253 & 0,9177 \\
\hline$[$ Fruta $] *[$ Fruta $]$ & 1 & 62,300 & 0,07 & 62,300 & 0,07 & 13,16 & 0,01 & 0,0016 & 0,9177 \\
\hline $\mathrm{pH}^{*}[$ Fruta $]$ & 1 & 4,080 & 14,08 & 4,080 & 14,08 & 0,86 & 2,08 & 0,3635 & 0,1638 \\
\hline Residuo & 21 & 99,400 & 142,06 & 4,730 & 6,76 & & & & \\
\hline $\begin{array}{l}\text { Total } \\
\text { (corregido) }\end{array}$ & 26 & 524,520 & 2172,74 & & & & & & \\
\hline $\begin{array}{l}\text { Modelo } \\
\text { Ajustado* }\end{array}$ & \multicolumn{5}{|c|}{$\mathrm{Sg}=17,444-0,844^{*}[$ Fruta $]+0,032^{*}[\text { Fruta }]^{2}$} & \multicolumn{4}{|c|}{$\mathrm{Sm}=15,778+1,011^{*}[$ Fruta $]+0,001 *[\text { Fruta }]^{2}$} \\
\hline$r^{2}$ & \multicolumn{5}{|c|}{$79,67 \%$} & \multicolumn{4}{|c|}{$92,31 \%$} \\
\hline
\end{tabular}

*[Fruta] representa contenido de pulpa de guayaba o jugo de maracuyá 
Los estadísticos $\mathrm{r}^{2}$ indican que el modelo así ajustado explica 79,67 \% y 92,31 \% la variabilidad de la solubilidad del cocristalizado de pulpa de guayaba y de jugo de maracuyá, respectivamente. Por tanto, la solubilidad de los cocristalizados depende sólo de la concentración de fruta, ejerciendo el efecto cuadrático de la fruta una mayor influencia sobre la solubilidad del cocristalizado que los efectos principales en la solubilidad de cocristalizados de pulpa de guayaba (Figura 4a). Sin embargo, para el cocristalizado de maracuyá tiene mayor incidencia los efectos principales (Figura $4 \mathrm{~b}$ ).

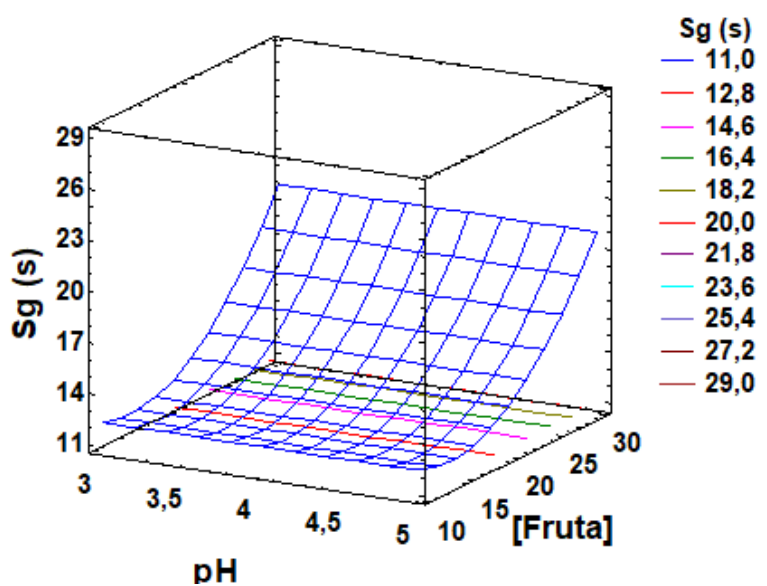

(a)

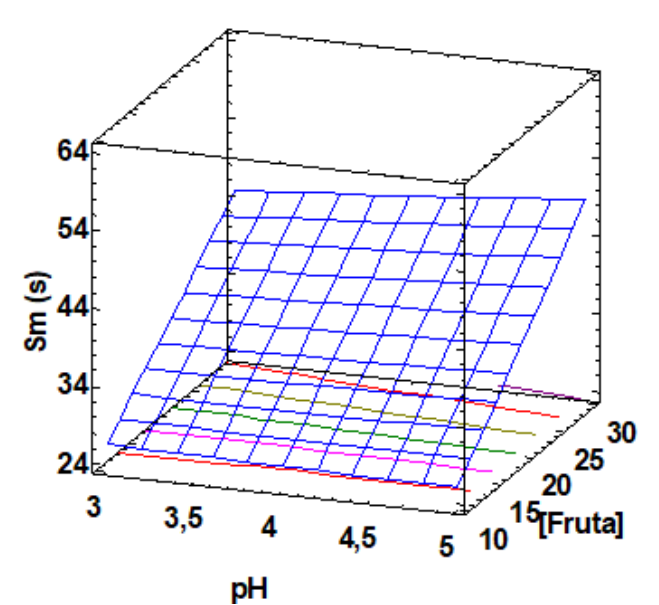

(b)

Figura 4. Solubilidad del cocristalizado, en función del pH y \% de pulpa de guayaba (a) y jugo de maracuyá (b) Fuente: elaboración propia.

El tiempo de solubilidad de cocristalizado de guayabas aumentó (solubilidad disminuyó) con el contenido de fruta (Figura 4) de forma exponencial, mientras el cocristalizado de maracuyá presentó un comportamiento casi lineal, por lo cual la solubilidad del cocristalizado de este último, aumento en menor proporción con el contenido de fruta. La máxima solubilidad (menor tiempo de solubilidad) del cocristalizado de guayaba, que se puede obtener según el modelo, fue de $11,91 \mathrm{~s}$, a pH de $4,4 \%$ y $13,1 \%$ de pulpa, y la de cocristalizado de jugo de maracuyá fue de $26,00 \mathrm{~s}$ a $\mathrm{pH}$ de $3,00 \%$ y 10,00 \% de jugo. Esto se explica por la menor densidad aparente del cocristalizado de pulpa de guayaba, y su porosidad, según sugiere [40], resultando que la solubilidad de las muestras podría, por tanto, estar más influenciada por la composición de la fruta y su proporción que por la azúcar refinada comercial [45].

El rango de solubilidad de cocristalizado de pulpa de guayaba $(11,67 \pm 1,15 \mathrm{~s}-22,00 \pm 5,57$ s), fueron similares a los de lo reportado por [40] para bebidas de cacao en polvo y el de jugo de maracuyá $(25,00 \pm 1,00 \mathrm{~s}-49,00 \pm 3,61 \mathrm{~s})$. Sin embargo, fueron menores que en cocristalizado de maracuyá comercial a $64^{\circ}$ Brix (37 s - 60 s) [39]. Esto influye en la reconstitución de un néctar $\mathrm{y}$, dado que a bajos tiempos de solubilidad se obtiene menor tiempo en la reconstitución de un néctar, se puede decir que los cristalizados presentan buenas propiedades de reconstrucción.

Ángulo de reposo: El ANOVA para el ángulo de reposo de los cocristalizados de pulpa de guayaba (Ag) y de jugo de maracuyá (Am) (Tabla 5) muestra que la interacción, al igual que los efectos principal y cuadrático de $\mathrm{pH}$, no presentaron diferencia significativa sobre los ángulos de reposo, dependiendo sólo de la concentración de la fruta en la mezcla.

Consecuentemente, se considera eliminar este factor de los modelos estadísticos. Los estadísticos $\mathrm{r}^{2}$ indican que el modelo así ajustado explica 97,92 \% y 98,10 \% la variabilidad 
del ángulo de reposo del cocristalizado de pulpa de guayaba y de jugo de maracuyá, respectivamente.

Tabla 5. Análisis de varianza para Ángulo de reposo de los productos cocristalizados Fuente: elaboración propia.

\begin{tabular}{|c|c|c|c|c|c|c|c|c|c|}
\hline \multirow[t]{2}{*}{ Fuente } & \multirow[t]{2}{*}{$\mathrm{Gl}$} & \multicolumn{2}{|c|}{ Suma de Cuadrados } & \multicolumn{2}{|c|}{ Cuadrado Medio } & \multicolumn{2}{|c|}{ Razón-F } & \multicolumn{2}{|c|}{ Valor-p } \\
\hline & & Guayaba & Maracuyá & Guayaba & Maracuyá & Guayaba & Maracuyá & Guayaba & Maracuyá \\
\hline $\mathrm{pH}$ & 1 & 0,02 & 0,010 & 0,02 & 0,01 & 0,04 & 0,00 & 0,8344 & 0,9618 \\
\hline [Fruta] & 1 & 426,52 & 12,453 & 426,52 & 12,45 & 1059,38 & 47,16 & 0,0000 & 0,0000 \\
\hline $\mathrm{pH}^{*} \mathrm{pH}$ & 1 & 0,73 & 0,010 & 0,73 & 0,01 & 1,82 & 0,03 & 0,1917 & 0,8650 \\
\hline$[\text { Fruta }]^{*}[$ Fruta $]$ & 1 & 9,31 & 2,920 & 9,31 & 2,92 & 23,12 & 11,05 & 0,0001 & 0,0032 \\
\hline $\mathrm{pH}^{*}[$ Fruta $]$ & 1 & 0,06 & 0,070 & 0,06 & 0,07 & 0,15 & 0,27 & 0,6995 & 0,6106 \\
\hline Residuo & 19 & 8,45 & 5,540 & 0,40 & 0,26 & & & & \\
\hline $\begin{array}{l}\text { Total } \\
\text { (corregido) }\end{array}$ & 26 & 445,09 & 298,110 & & & & & & \\
\hline $\begin{array}{l}\text { Modelo } \\
\text { Ajustado* }\end{array}$ & & $\begin{array}{r}\mathrm{Ag}=33,5 \\
0,\end{array}$ & $\begin{array}{l}27-0,011^{*} \\
012^{*}[\text { Fruta }\end{array}$ & Fruta] + & & $=31,209+$ & $0,680 *[$ Frut & $\mathrm{ta}]-0,007^{*}$ & {$[\text { Fruta }]^{2}$} \\
\hline
\end{tabular}

*[Fruta] representa contenido de pulpa de guayaba o jugo de maracuyá.

Según [43] y [46], una manera de caracterizar el comportamiento de polvos o gránulos durante el flujo es midiendo el ángulo de reposo, para lo cual los polvos que exhiben ángulos de reposo menor a $35^{\circ}$ son polvos no cohesivos y generalmente presentan propiedades de flujo libre, mientras que los que exhiben ángulos de reposo mayores a $45^{\circ}$ presentan comportamiento de polvos cohesivos, por tanto se puede afirmar que los cocristalizados de guayaba presentan propiedades de flujo libre a concentraciones de pulpa menores de $20 \%$, mientras que los cocristalizados de maracuyá a concentraciones de jugo por debajo de $10 \%$ (Figura 5). Es de anotar que el rango de los ángulos de reposo observado dependen en gran medida de la matriz (azúcar refinada comercial) que posee ángulos de reposo entre $31^{\circ}$ a $38^{\circ}$ [47].

El menor ángulo de reposo del cocristalizado de guayaba que se puede obtener, según el modelo, fue de $34,66^{\circ}$ a $\mathrm{pH}$ de 3,0 y $10,0 \%$ de pulpa, y la de cocristalizado de jugo de maracuyá fue de $37,31^{\circ}$ a $\mathrm{pH}$ de 3,0 y $10,0 \%$ de jugo.

El rango de ángulos de reposo de cocristalizado de pulpa de guayaba $\left(34,29 \pm 0,44^{\circ}-44,53\right.$ $\left.\pm 0,65^{\circ}\right)$ y el de jugo de maracuyá $\left(37,05 \pm 0,58^{\circ}-45,60 \pm 0,32^{\circ}\right)$ coinciden con los reportados para cocristalizado de limón $\left(26,3^{\circ}-30,6^{\circ}\right)$ [39] y para cocristalizado de maracuyá $\left(27^{\circ}-36^{\circ}\right)$ [48]. Sin embargo, presentó valores por debajo de los reparados para encapsulados por aspersión con base a lactosuero y pulpa de mango [36]. 


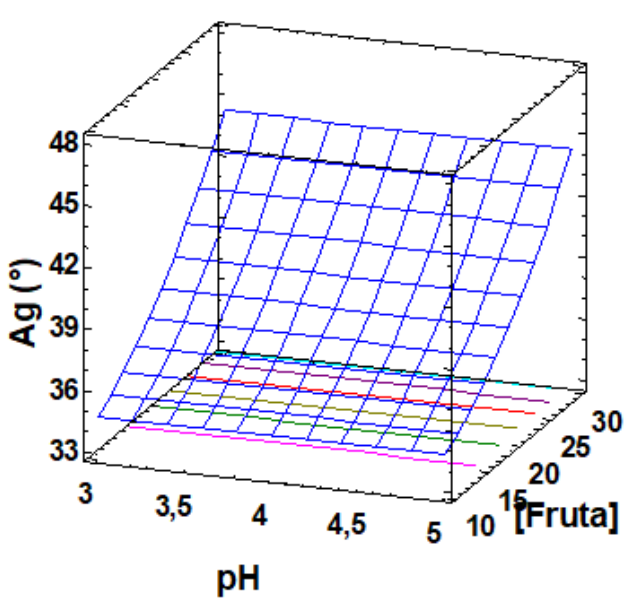

(a)

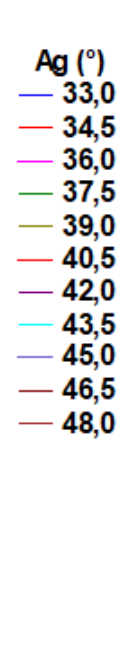

$\mathrm{Ag}\left({ }^{\circ}\right)$

$-34,5$

$-36,0$

$-37,5$

$-40,5$

$-42,0$

$-43,5$

$-46,5$

48,0

Figura 5. Ángulo de reposo del cocristalizado, en función del pH y \% de pulpa de guayaba (a) y jugo de maracuyá (b). Fuente: elaboración propia.

Higroscopicidad del cocristalizado: El ANOVA para la higroscopicidad del cocristalizado de pulpa de guayaba (Hig) y de jugo de maracuyá (Him) (Tabla 6) muestra que la interacción no presentó diferencia significativa (con un nivel de confianza del 95,0 \%), al igual que los efectos principal y cuadrático de $\mathrm{pH}$. Consecuentemente, se considera eliminar este factor de los modelos estadístico. Los estadísticos $\mathrm{r}^{2}$ indican que el modelo así ajustado explica 92,28 \% y 97,37 \% la variabilidad de la higroscopicidad del cocristalizado de pulpa de guayaba y de jugo de maracuyá, respectivamente, por lo que la higroscopicidad de los cocristalizados depende sólo de la concentración de fruta, ejerciendo el efecto cuadrático de la concentración de fruta una mayor influencia sobre la humedad del cocristalizado de guayaba (que los efectos principales (Figura 6a). Sin embargo, para el cocristalizado de maracuyá tiene mayor incidencia los efectos principales (Figura $6 \mathrm{~b}$ ).

Tabla 6. Análisis de varianza para Higroscopicidad de los productos cocristalizados

Fuente: elaboración propia.

\begin{tabular}{|c|c|c|c|c|c|c|c|c|c|}
\hline \multirow[t]{2}{*}{ Fuente } & \multirow[t]{2}{*}{ Gl } & \multicolumn{2}{|c|}{ Suma de Cuadrados } & \multicolumn{2}{|c|}{ Cuadrado Medio } & \multicolumn{2}{|c|}{ Razón-F } & \multicolumn{2}{|c|}{ Valor-P } \\
\hline & & Guayaba & Maracuyá & Guayaba & Maracuyá & Guayaba & Maracuyá & Guayaba & Maracuyá \\
\hline $\mathrm{pH}$ & 1 & 8,00 & 0,89 & 8,00 & 0,89 & 2,39 & 1,54 & 0,1367 & 0,2288 \\
\hline [Fruta] & 1 & 826,89 & 544,50 & 826,89 & 544,5 & 247,54 & 941,25 & 0,0000 & 0,0000 \\
\hline $\mathrm{pH}^{*} \mathrm{pH}$ & 1 & 0,07 & 0,30 & 0,07 & 0,30 & 0,02 & 0,51 & 0,8830 & 0,4821 \\
\hline$[$ Fruta $] *[$ Fruta $]$ & 1 & 124,52 & 0,02 & 124,52 & 0,02 & 37,28 & 0,03 & 0,0000 & 0,8597 \\
\hline $\mathrm{pH}^{*}[$ Fruta $]$ & 1 & 1,33 & 1,33 & 1,33 & 1,33 & 0,40 & 2,30 & 0,5343 & 0,1439 \\
\hline Residuo & 21 & 70,15 & 12,15 & 3,34 & 0,58 & & & & \\
\hline Total (corregido) & 26 & 1030,96 & 559,19 & & & & & & \\
\hline Modelo Ajustado* & \multicolumn{5}{|c|}{ Hig $=14,222+2,500[$ Fruta $]-0,045^{*}[\text { Fruta }]^{2}$} & \multicolumn{4}{|c|}{ Him $=34,556-0,528^{*}[$ Fruta $]-0,001[\text { Fruta }]^{2}$} \\
\hline $\mathrm{r}^{2}$ & \multicolumn{5}{|c|}{$92,28 \%$} & \multicolumn{4}{|c|}{$97,37 \%$} \\
\hline
\end{tabular}

*[Fruta] representa contenido de pulpa de guayaba o jugo de maracuyá.

La higroscopicidad del cocristalizado de pulpa de guayaba aumentó con la concentración de fruta (Figura 6a). Según [49], la sacarosa, en estado puro, tiende a adsorber agua a partir de una actividad de agua de 0,8, mientras que la fructosa y mezclas de azúcares tienen capacidad de adsorber agua a actividad de agua más baja que la sacarosa pura, eso explica la 
mayor higroscopicidad observada en las muestras con mayor proporción de pulpa (que contiene azúcares reductores), dado que la actividad acuosa del cocristalizado de pulpa de guayaba estuvo entre $0,37 \pm 00$ a $0,49 \pm 0,01$ (datos no mostrados). Sin embargo, el cocristalizado de jugo de maracuyá disminuye con el contenido de fruta (Figura 6b), esto se puede explicar porque los sólidos solubles del jugo favorecen la formación de cristales menos porosos, predominando este fenómeno sobre las características de absorción [35]. La actividad acuosa del cocristalizado de jugo de maracuyá estuvo entre 0,31 \pm 03 a 0,49 $\pm 0,00$ (datos no mostrados).

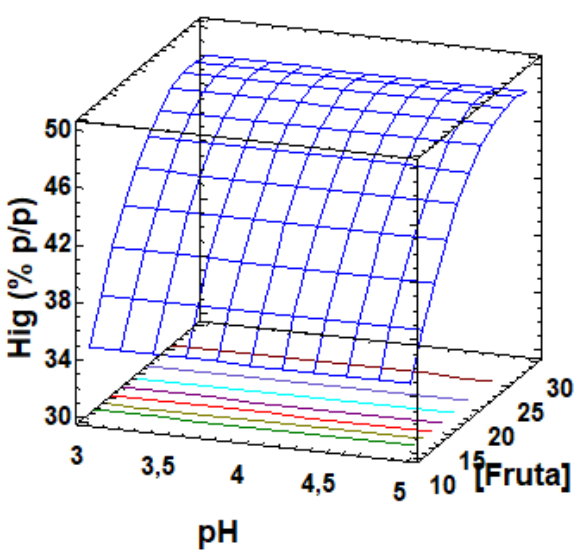

(a)

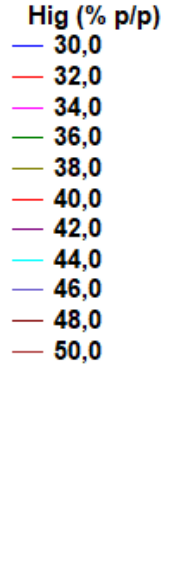

Figura 6 Higroscopicidad del cocristalizado. en función del pH y \% de pulpa de guayaba (a) y jugo de maracuyá

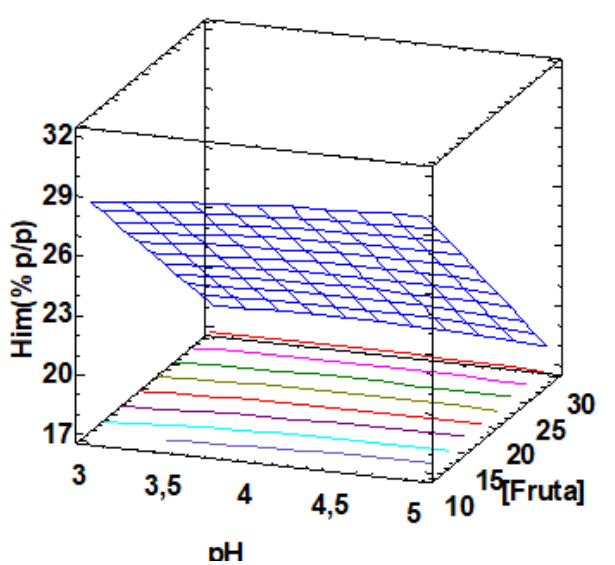

(b) (b). Fuente: elaboración propia.

La menor higroscopicidad del cocristalizado de guayaba que se puede obtener según el modelo, fue de $34,67 \% \mathrm{p} / \mathrm{p}$ a $\mathrm{pH}$ de $3,0 \%$ y 30,0 \% de pulpa, y la de cocristalizado de jugo de maracuyá $(\mathrm{Hm})$ fue de $18,22 \%$ p/p a pH de 3,0 y 30,0 \% de jugo.

El rango de higroscopicidad de cocristalizado de pulpa de guayaba $(33,67 \pm 2,08 \%-48,67$ $\pm 1,15 \%)$ p/p y el de jugo de maracuyá $(18,00 \pm 1,00 \%-30,00 \pm 0,00 \%)$ p/p, presentando valores superiores a los reparado para encapsulados por aspersión con base a lactosuero y pulpa de mango $(11,55 \%$ - 16,83\%) p/p [36], en microencapsulado de chirimoya $(4,76 \%$ $9,83 \%) \mathrm{p} / \mathrm{p}$ [38] y polvo de mango secado al vacío [50].

\subsection{Cinética de cristalización}

En la Figura 7 se observa el comportamiento en proceso de cocristalización para los cocristalizados de pulpa de guayaba y jugo de maracuyá a diferentes concentraciones de fruta y $\mathrm{pH}$ de 3,0, dado que fue el que tuvo la mayor velocidad de cristalización (gráficos no mostrados), y este no presentó diferencia significativa en las propiedades de los cocristalizadas. En la Figura 7 se evidencia un rango de una temperatura de nucleación máxima para los cocristalizados de $42,3{ }^{\circ} \mathrm{C}$ a $46,0^{\circ} \mathrm{C}$, donde se obtiene el máximo de viscosidad compleja en un rango de 7,9 Pa.s a 9,17 Pa.s para los cocristalizados de pulpa de guayaba y de jugo de maracuyá. A partir de estos rangos de temperatura, la viscosidad compleja comienza a descender indicando la formación y precipitación de los de cristales. 


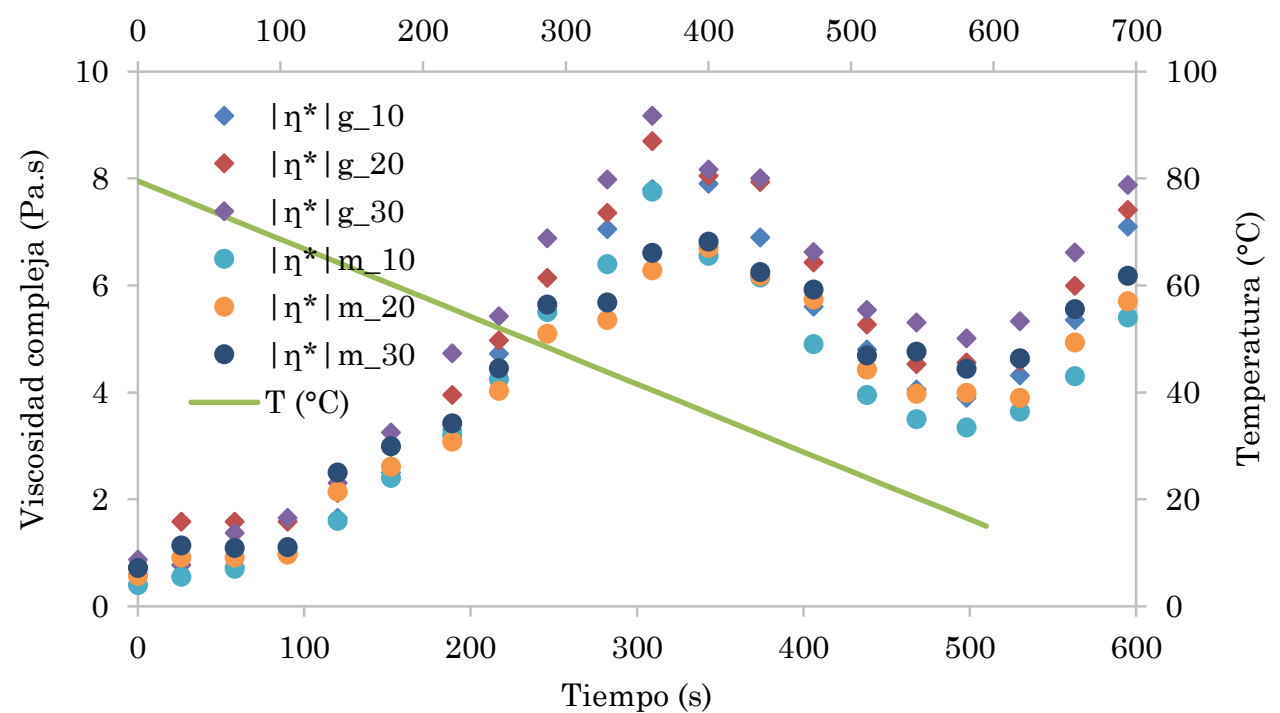

Figura 7. Temperatura y viscosidad compleja cocristalizado de guayaba $\left|\mathrm{n}^{*}\right|_{\mathrm{g}}$ y maracuyá $|\mathrm{n} *|_{\mathrm{m}}$, a lo largo del tiempo, a pH de 3,0, en el proceso de cocristalización. Fuente: elaboración propia.

En la Figura 8 se muestra la relación entre la viscosidad compleja del sistema a lo largo del tiempo de cristalización y la viscosidad compleja inicial del sistema $\left(\left|\eta^{*}\right| /\left|\eta^{*}\right|_{0},\right)^{a}$ diferentes concentraciones de fruta. Se observa, en general, que al principio del proceso (hasta $150 \mathrm{~s}$ ) el comportamiento cinético es similar a lo largo del tiempo, coincidiendo las curvas en el tiempo. Sin embargo, a medida que el proceso avanza, las viscosidades observadas comienzan a diferir entre sí, siendo influenciado por la concentración de fruta en el proceso, disminuyendo la relación de viscosidad compleja con la mayor concentración de fruta, presentándose una mayor diferencia en los cocristalizados de jugo de maracuyá. Es posible que esta disminución de viscosidad compleja se deba a que la pulpa de guayaba incide más que el jugo de maracuyá, por el tamaño de partícula, en la cristalización de la sacarosa, comportándose como impurezas.

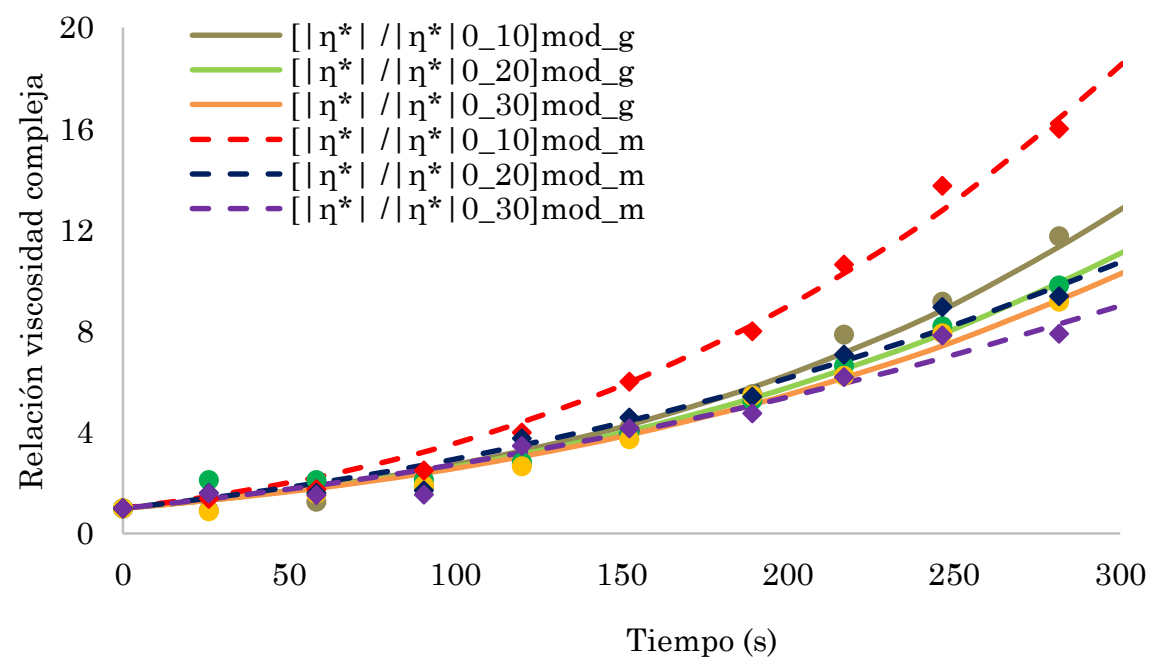

Figura 8. Tiempo y viscosidad compleja de cocristalizado de guayaba $\left|\mathrm{n}^{*}\right|_{\mathrm{g}}$ y maracuyá $|\mathrm{n} *|_{\mathrm{m}}$, a $\mathrm{pH}$ de 3,00 y [fruta] de $10 \%$, a lo largo del tiempo en el proceso de cocristalización. Fuente: elaboración propia. 
Los valores de a aumentaron con la concentración de fruta utilizadas en el proceso de cocristalización (Tabla 7), obteniéndose valores mayores en el cocristalizado de jugo de maracuyá que en el de pulpa de guayaba, indicando que la viscosidad compleja del cocristalizado es favorecida con el aumento de la concentración de fruta en el proceso de cocristalización, siendo esta mayor en los cocristalizado de jugo de maracuyá que en los de pulpa de guayaba.

El modelo propuesto por [26] explica entre un 99,78 \% y 99,5\% a (Tabla 7) los datos experimentales de la viscosidad compleja a través del tiempo en el proceso de cocristalización. Valores de $B$ superiores indican que la velocidad de cristalización aumente con el tiempo.

Todos los valores de $B$ del cocristalizado de guayaba fueron mayores que los de maracuyá, por lo tanto, se presentó una mayor velocidad de cristalización para los cristalizados de pulpa de guayaba que en los de maracuyá para el rango de concentración de fruta. Además, se puede observar que a medida que aumenta de concentración de fruta en el proceso de cristalización disminuye el valor de 6 , lo que indica que la velocidad de cocristalización se ve favorecida por la menor concentración de fruta, coincidiendo con lo reportado por [51]. Esto se puede explicar porque la incorporación de la sacarosa en el jugo permite una mayor densidad de nucleación, determinada por un tiempo de nucleación más corto [26].

Tabla 7. Parámetros a y 8 de la Ecuación (8) y coeficiente de determinación $\left(\mathrm{r}^{2}\right)$ para los cocristalizados de pulpa de guayaba y jugo de maracuyá a diferentes concentraciones de fruta utilizadas en proceso de cocristalización, a un $\mathrm{pH}$ de 3,0. Fuente: elaboración propia.

\begin{tabular}{cccc}
\hline Parámetros & $\alpha \times 10^{-2}\left(\mathrm{~s}^{-1}\right)$ & $B$ & $\mathrm{r}^{2}$ \\
\hline Cocristalizado de guayaba $10^{\circ}$ Brix & 2,33 & 4,81 & 0,9950 \\
Cocristalizado de guayaba $20^{\circ}$ Brix & 2,84 & 3,90 & 0,9974 \\
Cocristalizado de guayaba $30^{\circ}$ Brix & 2,99 & 3,64 & 0,9969 \\
\hline Cocristalizado de maracuyá $10^{\circ}$ Brix & 4,71 & 3,31 & 0,9978 \\
Cocristalizado de maracuyá $20^{\circ}$ Brix & 6,02 & 2,30 & 0,9938 \\
Cocristalizado de maracuyá $30{ }^{\circ}$ Brix & 6,20 & 2,09 & 0,9915 \\
\hline
\end{tabular}

\section{CONCLUSIONES}

El pH no afecta las propiedades de los cocristalizados y la concentración de fruta en el proceso de cocristalización debe ser lo más bajo posible, siempre que garantice la funcionalidad del producto, ya que, al aumentar la concentración de fruta añadida en el proceso, aumenta el tiempo de cristalización, aumenta la humedad final del producto y disminuye su solubilidad.

El azúcar en el jugo de maracuyá se incorporó de mejor forma que en la pulpa de guayaba, siendo la densidad aparente del cocristalizados de maracuyá mayor y la higroscopicidad, menor al de la pulpa de guayaba.

El modelo propuesto por [26] explica satisfactoriamente la cinética de cristalización de la pulpa de guayaba y de jugo de maracuyá. La velocidad de cocristalización se ve favorecida por la menor concentración de fruta y se pudo observar que se presentó una mayor velocidad de cristalización para los cristalizados de pulpa de guayaba que en los cristalizados de jugo de maracuyá. La nucleación máxima para los cocristalizados se presentó en el rango de $42,3{ }^{\circ} \mathrm{C}$ a $46,0^{\circ} \mathrm{C}$. 


\section{AGRADECIMIENTOS}

Los autores agradecen a la Universidad del Atlántico, por el aporte de laboratorios para análisis físicos y químicos de las pulpas y parte de la caracterización física de los cocristalizados; de igual manera a la Universidad de Córdoba, por su aporte de laboratorios para elaboración de cristalizados y análisis de necesarios para cinética de cristalización.

El artículo no contó con el apoyo económico de algún proyecto ni de alguna agencia financiadora pública o privada.

\section{CONFLICTOS DE INTERÉS}

Los autores declaramos no tener conflictos de interés ni financiero ni profesional ni personal que pueda influir de forma inapropiada en los resultados obtenidos o las interpretaciones propuestas.

\section{CONTRIBUCIÓN DE LOS AUTORES}

Tanto la concepción como la redacción y análisis de la información fue realizada de manera conjunta por los autores.

Ramiro Torres-Gallo, además, contribuyó sustancialmente al proponer la orientación de la temática, y diseño metodológico de búsqueda y la estructuración y análisis de resultados.

Teófilo Arteaga-Márquez participó en la compilación de los artículos y en la realización de análisis de laboratorio.

Everardo Montes-Montes participó en la definición del alcance del artículo, así como en el análisis y las conclusiones.

\section{REFERENCIAS}

[1] L. Carcamo-Medina; M. Eleazar-Turcios; L. E. Ordoñez-Santos, "Cambios en los compuestos bioactivos del jugo de uchuva (Physalis peruviana L.) pasteurizado,” TecnoLógicas, vol. 22, no. 45, pp. 145-155, May. 2019. https://doi.org/10.22430/22565337.1279

[2] M. De Souza Silva; E. Ataíde Machado; A. K. E. dos Santos; J. M. Azevedo Souza, "Qualidade de frutos de maracujazeiro amarelo produzidos na safra e entressafra no vale do São Francisco," Rev. Iberoam. Tecnol. Postcosecha, vol. 17, no. 1, pp. 41-49, 2016, Dec., 2019. URL

[3] L. E. Ordóñez-Santos; A. Vázquez-Riascos, "Effect of processing and storage time on the vitamin C and lycopene contents of nectar of pink guava (Psidium guajava L.)," Arch. Latinoam. Nutr., vol. 60, no. 3, pp. 280-284, 2010. URL

[4] J. Aguiar; B. N. Estevinho; L. Santos, "Microencapsulation of natural antioxidants for food application The specific case of coffee antioxidants - A review," Trends Food Sci. Technol., vol. 58, pp. 21-39, Dec. 2016. https://doi.org/10.1016/j.tifs.2016.10.012

[5] G. Lozano-Vazquez; C. Lobato-Calleros; H. Escalona-Buendia; G. Chavez; J. Alvarez-Ramirez; E. J. VernonCarter, "Effect of the weight ratio of alginate-modified tapioca starch on the physicochemical properties and release kinetics of chlorogenic acid containing beads," Food Hydrocoll., vol. 48, pp. 301-311, Jun. 2015. https://doi.org/10.1016/j.foodhyd.2015.02.032

[6] A. G. Fernandes; G. Matias dos Santos; D. Sales da Silva; P. H. Machado de Sousa; G. Arraes Maia; R. Wilane de Figueiredo, "Chemical and physicochemical characteristics changes during passion fruit juice processing," Ciência e Tecnol. Aliment., vol. 31, no. 3, pp. 747-751, Sep. 2011. URL

[7] V. Stojceska; J. Atuonwu; S. A. Tassou, "Ohmic and conventional drying of citrus products: Energy efficiency, greenhouse gas emissions and nutritional properties," Energy Procedia, vol. 161, pp. 165-173, Mar. 2019. https://doi.org/10.1016/j.egypro.2019.02.076 
[8] G. Ozkan; P. Franco; I. De Marco; J. Xiao; E. Capanoglu, "A review of microencapsulation methods for food antioxidants: Principles, advantages, drawbacks and applications," Food Chem., vol. 272, pp. 494-506, Jan. 2019. https://doi.org/10.1016/j.foodchem.2018.07.205

[9] J. Castro-Rosas et al., "Recent advances in microencapsulation of natural sources of antimicrobial compounds used in food - A review," Food Res. Int., vol. 102, pp. 575-587, Dec. 2017. https://doi.org/10.1016/j.foodres.2017.09.054

[10] X. Luo; J. Sedman; A. Ismail, "Microencapsulation of oregano ( Origanum vulgare L .), rosemary (Rosmarinus officinalis L .) and sage ( Salvia officinalis L.) essential oils in B - lactoglobulin," J. Food Sci. Technol., vol. 4, no. 9, pp. 970-985, Dec. 2019. https://doi.org/10.25177/JFST.4.9.RA.612

[11] A. López-Córdoba; L. Gallo; V. Bucalá; M. Martino; A. Navarro, "Co-crystallization of zinc sulfate with sucrose: A promissory strategy to render zinc solid dosage forms more palatable," J. Food Eng., vol. 170, pp. 100-107, Feb. 2016. https://doi.org/10.1016/j.jfoodeng.2015.09.024

[12] A. López-Córdoba; L. Deladino; L. Agudelo-Mesa; M. Martino, "Yerba mate antioxidant powders obtained by co-crystallization: Stability during storage," J. Food Eng., vol. 124, pp. 158-165, Mar. 2014. https://doi.org/10.1016/j.jfoodeng.2013.10.010

[13] B. R. Sardar; R. S. Singhal, "Characterization of co-crystallized sucrose entrapped with cardamom oleoresin," J. Food Eng., vol. 117, no. 4, pp. 521-529, Aug. 2013. https://doi.org/10.1016/j.jfoodeng.2012.12.011

[14] A. López-Córdoba; S. Matera; L. Deladino; A. Hoya; A. Navarro; M. Martino, "Compressed tablets based on mineral-functionalized starch and co-crystallized sucrose with natural antioxidants," J. Food Eng., vol. 146, pp. 234-242, Feb. 2015. https://doi.org/10.1016/j.jfoodeng.2014.09.019

[15] R. D. Andrade; K. Blanquicett; R. D. Rangel, "Efecto del pH, sólidos solubles y zumo adicionado sobre el color y la vitamina C de zumo de naranja agria cocristalizado," Inf. Tecnol., vol. 27, no. 6, pp. 129-134, 2016. http://dx.doi.org/10.4067/S0718-07642016000600013

[16] B. R. Bhandari; N. Datta; B. R. D'Arcy; G. B. Rintoul, "Co-crystallization of honey with sucrose," $L W T$ Food Sci. Technol., vol. 31, no. 2, pp. 138-142, Mar. 1998. https://doi.org/10.1006/fstl.1997.0316

[17] C. I. Beristain; A. Vazquez; H. S. Garcia; E. J. Vernon-Carter, "Encapsulation of orange peel oil by cocrystallization," LWT - Food Sci. Technol., vol. 29, no. 7, pp. 645-647, Nov. 1996. https://doi.org/10.1006/fstl.1996.0098

[18] K. Shimono; K. Kadota; Y. Tozuka; A. Shimosaka; Y. Shirakawa; J. Hidaka, "Kinetics of co-crystal formation with caffeine and citric acid via liquid-assisted grinding analyzed using the distinct element method," Eur. J. Pharm. Sci., vol. 76, pp. 217-224, Aug. 2015. https://doi.org/10.1016/j.ejps.2015.05.017

[19] C. I. Beristain; R. E. Mendoza; H. S. Garcia; A. Vazquez, "Cocrystallization of Jamaica (Hibiscus sabdarifa L.) granules.," LWT. Food Sci. Technol., vol. 27, no. 4, pp. 347-349, Aug. 1994. https://doi.org/10.1006/fstl.1994.1071

[20] M. W. Okoth; A. R. Kaahwa; J. K. Imungi, "The effect of homogenisation, stabiliser and amylase on cloudiness of passion fruit juice," Food Control, vol. 11, no. 4, pp. 305-311, Aug. 2000. https://doi.org/10.1016/S0956-7135(99)00107-3

[21] L. B. Quast; S. G. Farina; E. Quast; M. A. Vieira; M. B. Queiroz, "Co-crystallized honey with sucrose: Evaluation of process and product characterization," J. Food Process. Preserv., vol. 44, no. 11, p. e14876, Nov. 2020. https://doi.org/10.1111/JFPP.14876

[22] S. M. J. Vieira; S. M. Couto; P. C. Corrêa; A. E. O. dos Santos; P. R. Cecom; D. J. P. da Silva, "Características físicas de goiabas (Psidium guajava L.) submetidas a tratamento hidrotérmico," Rev. Bras. Eng. Agrícola Ambient., vol. 12, no. 4, pp. 408-414, Ago. 2008. https://doi.org/10.1590/S1415-43662008000400012

[23] I. M. del P. Pinzón; G. Fischer; G. Corredor, "Determinación de los estados de madurez del fruto de la gulupa (Passiflora edulis Sims.)," Agron. Colomb., vol. 25, no. 1, pp. 83-95, Jan. 2007. URL

[24] D. F. Tirado; P. M. Montero; D. Acevedo, "Estudio Comparativo de Métodos Empleados para la Determinación de Humedad de Varias Matrices Alimentarias," Inf. tecnológica, vol. 26, no. 2, pp. 3-10, 2015. http://dx.doi.org/10.4067/S0718-07642015000200002

[25] Y. Z. Cai; H. Corke, "Production and properties of spray-dried Amaranthus betacyanin pigments," J. Food Sci., vol. 65, no. 7, pp. 1248-1252, Jul. 2000. https://doi.org/10.1111/j.1365-2621.2000.tb10273.x

[26] K. D. Kim; S. H. Lee; H. K. Ahn, "Observation of nucleation effect on crystallization in lithium aluminosilicate glass by viscosity measurement," J. Non. Cryst. Solids, vol. 336, no. 3, pp. 195-201, May. 2004. https://doi.org/10.1016/j.jnoncrysol.2004.01.001

[27] H. G. Cuastumal Canacuan; B. L. Valencia Murillo; L. E. Ordóñez Santos, "Effects of heat treatment on the concentration of vitamin C and surface color in three tropicale fruits," Rev. Lasallista Investig., vol. 13, no. 1, pp. 85-93, 2016. http://dx.doi.org/10.22507/RLI.V13N1A7

[28] D. Rojas-Barquera; C.-E. Narváez-Cuenca, "Determinación de vitamina C, compuestos fenólicos totales y actividad antioxidante de frutas de guayaba (Psidium guajava L.) cultivadas en Colombia," Quim. Nova, vol. 32, no. 9, pp. 2336-2340, 2009. https://doi.org/10.1590/S0100-40422009000900019 
[29] R. D. Andrade et al., "Physicochemical and rheological characterization of guava pulp (Psidium guajava L.) varieties hybrid Klom Sali, Puerto Rico, D14 and Red,” Vitae, vol. 16, no. 1, May. 2009. URL

[30] N. Laguado et al., "Efecto de la fertilización y del estado de madurez sobre la calidad de frutos de guayaba (Psidium guajava L.)," Rev. la Fac. Agron. la Univ. del Zulia, vol. 12, no. 4, May. 2012. URL

[31] C. Granados Conde; K. P. Tinoco Guardo; E. Granados Llamas; N. P. Pájaro-Castro; Y. García Milano, "Caracterización química y evaluación de la actividad antioxidante de la pulpa de Passiflora edulis Sims (gulupa)”, Rev. Cuba. Plantas Medic., vol. 22, no. 2, pp. 1-10, 2017. URL

[32] A. L. López; N. L. Matute; A. P. Echavarría, "Evaluación fisicoquimica y capacidad antioxidante de Moringa (Moringa oleífera) Y Maracuyá (Passiflora edulis,” Rev. Cumbres, vol. 4, no. 1, pp. 35-42, 2017. URL

[33] J. De la Cruz; M. Vargas; O. Del Angel; H. S. Garcia, "Estudio de las características sensoriales, fisicoquímicas y fisiológicas en fresco y durante el almacenamiento refrigerado de maracuyá amarillo (Passiflora edulis Sims var. flavicarp a, Degener), para tres cultivares de Veracruz, México.," Rev. Iberoam. Tecnol. Postcosecha, vol. 11, no. 2, pp. 130-142, Jan. 2010. URL

[34] C. W. Wilson; P. E. Shaw; C. W. Campbell, "Determination of organic acids and sugars in guava (Psidium guajava L.) cultivars by high-performance liquid chromatography," J. Sci. Food Agric., vol. 33, no. 8, pp. 777-780, Aug. 1982. https://doi.org/10.1002/JSFA.2740330815

[35] R. D. Andrade Pizarro; K. Blanquicett González; R. Rangel Terraza, "Propiedades físicas de naranja agria cocristalizada: efecto del pH, sólidos solubles y zumo adicionado," Agron. Mesoam., vol. 28, no. 2, pp. 427437, 2017. URL

[36] F. A. Mendoza-Corvis; M. Arteaga M.; O. Pérez S., "Comportamiento de la vitamina C en un producto a base de lactosuero y pulpa de mango variedad Magdalena River (Mangífera indica /.) durante el secado por aspersión," Rev. Chil. Nutr., vol. 43, no. 2, pp. 159-166, Jun. 2016. https://doi.org/10.4067/S071775182016000200008

[37] L. Naddaf; B. Avalo; M. Oliveros, "Secado por aspersión de jugo natural de naranja utilizando los encapsulantes maltodextrina y goma arábiga,” Rev. Tec. la Fac. Ing. Univ. del Zulia, vol. 35, no. 1, pp. 2027, Abr. 2012. URL

[38] C. Rivas Reyes, "Microencapsulación y estabilización enzimática del jugo de chirimoya (Annona cherimola Mill)", Tesis de maestría, Inst. Polit. Nal., 2010. URL

[39] Z. Astolfi Filho, "Encapsulação de sucos de frutas por co-cristalização com sacarose,", (Tesis de Maestría), Univ. Est. Paulista, 2003. URL

[40] T. A. Shittu; M. O. Lawal, "Factors affecting instant properties of powdered cocoa beverages," Food Chem., vol. 100, no. 1, pp. 91-98, Jan. 2007. https://doi.org/10.1016/j.foodchem.2005.09.013

[41] P. Cerezal Mezquita; A. Carrasco Verdejo; K. Pinto Tapia; R. Arcos Zavala, "Suplemento alimenticio de alto contenido proteico para niños de 2 - 5 años. II. Propiedades físicas, químicas, reológicas y color,” Interciencia, vol. 33, no. 4, pp. 301-307, Abr. 2008. URL

[42] C. Sáenz; E. Sepúlveda; N. Pak; X. Vallejos, "Uso de fibra dietética de nopal en la formulación de un polvo para flan,” ALAN, vol. 52, no. 4, pp. 387-392, Dec. 2002. URL

[43] M. Peleg; N. Pollak, "The problem of equilibrium conditions in stress relaxation analyses of solid foods," $J$. Texture Stud., vol. 13, no. 1, pp. 1-11, Mar. 1982. https://doi.org/10.1111/j.1745-4603.1982.tb00873.x

[44] G. V. Barbosa-Cánovas; H. Vega-Mercado; A. Ibarz Ribas, Deshidratación de alimentos. Zaragoza: Acribia, 2000.

[45] A. A. Abdalla; M. A. Mohammed; H. A. Mudawi, "Production and Quality Assessment of Instant Baobab (Adansonia digitata L.)," Adv. J. Food Sci. Technol., vol. 2, no. 2, pp. 125-133, Jan. 2010. URL

[46] A. López-Córdoba; A. Navarro, "Physicochemical properties and stability of sucrose/glucose agglomerates obtained by cocrystallization," J. Food Process Eng., vol. 41, no. 8, e12901, Oct. 2018, https://doi.org/10.1111/JFPE.12901

[47] L. Deladino; P. S. Anbinder; A. S. Navarro; M. N. Martino, "Co-crystallization of yerba mate extract (Ilex paraguariensis) and mineral salts within a sucrose matrix," J. Food Eng., vol. 80, no. 2, pp. 573-580, May. 2007. https://doi.org/10.1016/j.jfoodeng.2006.06.016

[48] E. Montes Montes; C. De Paula; F. Ortega, "Determinación de las condiciones óptimas de encapsulamiento por co-cristalización de jugo de maracuyá (Passiflora edulis)," Temas Agrar., vol. 12, no. 1, pp. 5-12, Jan. 2007. https://doi.org/10.21897/rta.v12i1.646

[49] J. H. Dittmar, "Hygroscopicity of Sugars and Sugar Mixtures," Ind. Eng. Chem., vol. 27, no. 3, pp. 333-335, Mar. 1935. https://doi.org/10.1021/IE50303A021

[50] S. Jaya; H. Das, "Effect of maltodextrin, glycerol monostearate and tricalcium phosphate on vacuum dried mango powder properties," J. Food Eng., vol. 63, no. 2, pp. 125-134, Jun. 2004. https://doi.org/10.1016/S0260-8774(03)00135-3

[51] Z. Astolfi-Filho; A. C. Souza; É. C. D. Reipert; V. R. N. Telis, "Encapsulação de suco de maracujá por cocristalização com sacarose: cinética de cristalização e propriedades físicas," Ciência e Tecnol. Aliment., vol. 25, no. 4, pp. 795-801, Dec. 2005. https://doi.org/10.1590/s0101-20612005000400027 